\title{
EVIDENNCIAS DE AÇÕES DE ENFERMAGEM \\ EM PROMOÇÃO DA SAÚDE PARA UM \\ ENVELHECIMENTO ATIVO: revisão Integrativa.
}

Cibelly Aliny Siqueira Lima Freitas ${ }^{1}$

Maria Josefina da Silva²

Neiva Francenely Cunha Vieira ${ }^{3}$

Lorena Barbosa Ximenes ${ }^{4}$

Maria da Conceição Coelho Brito ${ }^{5}$

Fabiane do Amaral Gubert ${ }^{6}$

resumo

Envelhecimento Ativo é o processo de otimização das oportunidades de saúde com o objetivo de aumentar a qualidade de vida do idoso. Para o enfermeiro, membro da equipe multidisciplinar, promover um cuidado gerontológico específico constitui atividade primordial. O objetivo da revisão integrativa é analisar as evidências disponíveis na literatura sobre as ações de Enfermagem em Promoção da Saúde para um Envelhecimento Ativo. Para a seleção dos artigos foram consultadas as bases de dados eletrônicas, Lilacs, Medline, Cochrane, Cinahl e Scopus; e ao total quatro artigos constituíram a amostra. Os materiais analisados apontam a Promoção da Saúde como essencial para o envelhecimento ativo e propõe a existência de uma relação entre cultura e saúde. Ao final, a presente revisão favorece a aquisição de conhecimentos acerca das ações de Enfermagem com ênfase na relação interpessoal entre enfermeiros/idosos, baseada na comunicação e princípios éticos.

\footnotetext{
1 Enfermeira. Doutoranda em Enfermagem da Universidade Federal do Ceará - UFC. Docente do curso de Graduação em Enfermagem da Universidade Estadual Vale do Acaraú. Sobral, Ceará, Brasil. E-mail: cibellyaliny@gmail.com

2 Enfermeira. Doutora em Enfermagem. Docente do Programa de Pós-graduação da UFC. Fortaleza, Ceará, Brasil. E-mail: mjosefina@terra.com.br

3 Enfermeira. PhD em Educação em Saúde. Professora Associada do Departamento de Enfermagem da UFC. Bolsista de Produtividade do CNPq. Fortaleza, Ceará, Brasil. E-mail: neivafrancenely@hotmail.com 4 Enfermeira, Doutora em Enfermagem, Docente do Programa de Pós-Graduação em Enfermagem da UFC. Fortaleza, Ceará, Brasil. E-mail: Ibximenes2005@yahoo.com.br

5 Discente do Curso de Enfermagem da Universidade Estadual Vale do Acaraú, Bolsista do Programa de Iniciação Científica da UVA. Sobral, Ceará, Brasil. E-mail: marycey@hotmail.com

6 Enfermeira, Doutoranda em Enfermagem da UFC, Bolsista CAPES, Fortaleza, Ceará, Brasil. E-mail: fabianegubert@hotmail.com
} 


\section{Introdução}

O envelhecimento da população é um fenômeno mundial iniciado, a princípio, nos países desenvolvidos. Fatores como a queda da mortalidade, urbanização adequada das cidades, melhoria nutricional, elevação dos níveis de higiene pessoal e ambiental, assim como, os avanços tecnológicos contribuíram para este cenário (SANTOS, 2008).

Nos países menos desenvolvidos como o Brasil, o aumento da expectativa de vida tem sido evidenciado pelos avanços tecnológicos relacionados à área de saúde nos últimos 60 anos, como o uso de vacinas, antibióticos e quimioterápicos, que tornaram possível a prevenção ou cura de muitas doenças. Aliado a esses fatores, tem-se a queda de fecundidade, iniciada na década de 60, que permitiu a ocorrência de uma grande explosão demográfica. Estimase que nos próximos 20 anos a população de idosos no Brasil poderá alcançar, e até mesmo ultrapassar, a cifra dos 30 milhões de pessoas, o que representará aproximadamente $13 \%$ da população (CAMARANO, 2002).

Essa realidade modifica a epidemiologia do País, que passa a trazer a consciência da existência da velhice como uma questão social. Questão essa, que pede grande atenção, pois está diretamente relacionada com crise de identidade, mudança de papéis, aposentadoria, perdas diversas e diminuição dos contatos sociais, além da ocorrência de doenças crônicas que são bastante comuns nessa fase da vida (OLIVEIRA et al.; 2005).

A magnitude do aumento dos custos da assistência à saúde, em função do envelhecimento da população, advém, em parte, da proporção de idosos com problemas crônicos (ou seja, com necessidades permanentes de atenção à saúde). Estima-se que entre $75 \%$ e $80 \%$ da população de 60 anos ou mais na América Latina, tem pelo menos uma doença crônica. Uma estimativa conservadora para o Brasil, aplicando essa proporção para o início do século, resultaria em algo como mais de 11 milhões de pessoas com 60 anos ou mais nessa condição. Isso representa um contingente que poderá aumentar para 27 milhões, em 2025, e para aproximadamente 50 milhões, em 2050 (PICCINI et al.; 2006).

Dadas as limitações do sistema de saúde pública brasileiro, o rápido processo de envelhecimento aponta para a necessidade de se redefinirem as políticas desse setor, com vistas à necessidade de geração de recursos e 
de construção de infra-estrutura que permitam um envelhecimento ativo (WONG e CARVALHO, 2006). De acordo com a Organização Pan-Americana da Saúde (2005), Envelhecimento Ativo é o processo de otimização das oportunidades de saúde, participação e segurança, com o objetivo de aumentar a expectativa de uma vida saudável e a qualidade de vida para todas as pessoas que estão envelhecendo, inclusive as que são frágeis, fisicamente incapacitadas e que requerem cuidados.

O Envelhecimento Ativo é sinônimo de uma vida saudável, participativa e com seguridade social. Para promovê-lo, os sistemas de saúde necessitam ter uma perspectiva de curso de vida que vise à promoção da saúde e o acesso eqüitativo ao cuidado primário e de longo prazo de qualidade (WORLD HEALTH ORGANIZATION, 2005).

O movimento da Promoção da Saúde (PS) é uma resposta a esses desafios, ao desenvolver ações voltadas ao envelhecimento saudável. O enfoque da Promoção da Saúde na velhice deve ser voltado ao bom funcionamento físico, mental e social, assim como à prevenção das enfermidades e incapacidades. Tal enfoque está proposto na Política Nacional do Idoso (PNI), que dispõe sobre: promover o envelhecimento saudável (envelhecimento ativo); manter e melhorar, ao máximo, a capacidade funcional dos idosos; prevenir as doenças; recuperar a saúde dos que adoecem; e reabilitar aqueles que venham a ter a sua capacidade funcional restringida, de modo a garantir-lhe a permanência no meio em que vivem, exercendo de forma independente suas funções na sociedade (LUNARDI et al.; 2009).

No que se refere ao papel do enfermeiro na promoção da saúde da pessoa idosa, esse contribui para construção da autonomia do idoso e empoderamento, bem como na apreensão de conhecimentos relacionados às particularidades desta população, priorizando as questões demográficas e epidemiológicas; diferenciando as alterações fisiológicas e patológicas no processo de envelhecimento. Outras atribuições podem ser relativas ao conhecimento da legislação nacional e políticas públicas voltadas às pessoas idosas, de forma à difundi-las entre os próprios idosos, família e comunidade. Ao desenvolver ações que considerem as limitações e a presença das Doenças Crônicas Não Transmissíveis (DCNT) possibilita a manutenção da sua autonomia e independência; capacitando as pessoas idosas, família, comunidade, estudantes, professores e trabalhadores acerca do processo de envelhecimento (BRASIL, 1998).

Na enfermagem, a Prática Baseada em Evidências, é uma abordagem que possibilita a melhoria da qualidade da assistência e incentiva o profissional de saúde a buscar conhecimento científico por meio do desenvolvi- 
mento de pesquisas ou aplicação na sua prática, dos resultados encontrados na literatura. Esse método pode ser direcionado para definição de conceitos, revisão de teorias ou análise metodológica de estudos acerca de um determinado objeto, contribuindo para o aprofundamento do conhecimento do tema investigado. Para este estudo, selecionou-se como método um dos recursos da Prática Baseada em Evidências: a revisão integrativa da literatura, a qual possibilita a síntese e a análise do conhecimento científico já produzido sobre o tema investigado (GALVÃO et al.; 2002).

Considerando que dentre as ações de promoção da saúde em enfermagem, voltadas para o cuidado da pessoa idosa, está a apropriação de conhecimentos sobre as peculiaridades que envolvem o Envelhecimento, justifica-se a realização de uma revisão integrativa. Tal revisão, além de permitir uma interpretação do conhecimento produzido na área, com o propósito de auxiliar no desenvolvimento de futuras investigações, contribui para a melhoria da atenção à saúde do idoso. Assim, este estudo tem como objetivo analisar as evidências disponíveis na literatura sobre as ações de Enfermagem em Promoção da Saúde para um Envelhecimento Ativo.

\section{Procedimentos Metodológicos}

Para condução desta investigação, adotou-se a revisão integrativa da literatura, visto que ela contribui para o processo de sistematização e análise dos resultados, visando à compreensão de um determinado tema a partir de outros estudos independentes (GALVÃO et al.; 2002). Na operacionalização da revisão, utilizaram-se as seguintes etapas: identificação do tema e seleção da questão norteadora; estabelecimento dos critérios para a seleção da amostra; definição das informações a serem extraídas dos estudos selecionados e categorização dos estudos; avaliação dos estudos incluídos na revisão integrativa e finalmente, a interpretação dos resultados e apresentação da revisão. Formulou-se a seguinte questão para guiar a revisão integrativa: Quais são as ações de enfermagem em Promoção da Saúde voltadas para o Envelhecimento Ativo?

O levantamento bibliográfico foi realizado no período de 01/09/09 a 30/09/09 nas bases de dados: Lilacs (Literatura Latino-Americana e do Caribe em Ciências da Saúde); Medline (Medical Literature Analysis and Retrieval Sistem on-line); Biblioteca Cochrane; Cinahl (Cumulative Index to Nursing and Allied Health Literature) e Scopus. Dessa forma, procurou-se ampliar o âmbito da pesquisa, minimizando possíveis vieses nessa etapa do processo de elaboração da revisão integrativa. Em virtude das características específicas para o acesso das bases de dados selecionadas, as estratégias utilizadas para 
localizar os artigos foram adaptadas, tendo como eixo norteador a pergunta e os critérios de inclusão do estudo.

Os critérios de inclusão dos artigos definidos foram: artigos disponíveis eletronicamente publicados no período de 1999 a 2009; nos idiomas inglês, português ou espanhol; e que abordam ações promotoras de saúde em Enfermagem voltada para o Envelhecimento saudável. Foram excluídos durante a busca: produção duplicada, editoriais, cartas ao editor e boletins epidemiológicos.

Os descritores foram selecionados a partir da terminologia em saúde consultada nos Descritores em Ciências da Saúde (DeCS). A busca nas referidas bases de dados foram realizadas a partir dos seguintes descritores: Promoção da Saúde, Enfermagem e Envelhecimento, nos três idiomas delimitados nos critérios de inclusão.

Na busca inicial, foram encontrados 18 artigos na base de dados Lilacs, sendo que nas demais bases nenhum artigo foi localizado. Dos materiais selecionados, por meio da leitura dos resumos disponíveis, excluiu-se 12 publicações. Ao final apenas quatro definiram a amostra final da presente revisão.

A coleta de informações se deu a partir de um instrumento proposto por Ursi (2005), em seus estudos sobre revisão integrativa, o qual foi preenchido para cada artigo da amostra final da revisão. O instrumento contempla os seguintes itens: identificação do artigo original; características metodológicas do estudo; e avaliação do rigor metodológico, das intervenções aplicadas e dos resultados, dentre outras informações.

A apresentação dos resultados e discussão dos dados foi realizada de forma descritiva e por meio da construção de dois quadros sinópticos contemplando os seguintes aspectos: nome da pesquisa; autores; delineamento; resultados e recomendações/conclusões.

\section{Resultados e Discussões}

Os quatro artigos selecionados foram publicados a partir do ano de 2006, o que mostra uma tendência de estudos na área ainda recente. Em relação às publicações, essas foram divulgadas em periódicos de Enfermagem geral, sendo duas na revista Texto \& Contexto-Enfermagem, e as demais nas revistas Acta Paulista de Enfermagem e Revista da Escola de Enfermagem da USP. Os autores correspondem em sua maioria a enfermeiros, docentes de cursos de graduação em enfermagem. Dentre os artigos, somente um contou com alunos de graduação em sua composição. Com isso observa-se que profissionais e alunos compreendem a enfermagem como uma ciência dinâmica, que necessita de fundamentos científicos que justifiquem sua prática. 
O artigo A, exposto no quadro a seguir, esboça as necessidades de saúde e de educação de cuidadores domiciliares de idosos. Isso evidencia que a falta de suporte e estrutura mais adequada não contribui para uma assistência mais efetiva ao idoso.

\begin{tabular}{|c|c|c|c|}
\hline Titulo do artigo & Metodologia & $\begin{array}{l}\text { Intervenção } \\
\text { estudada }\end{array}$ & $\begin{array}{l}\text { Recomendações/ } \\
\text { Conclusões }\end{array}$ \\
\hline $\begin{array}{c}\text { Artigo A - Necessida- } \\
\text { des de educação em } \\
\text { saúde dos cuidadores } \\
\text { de pessoas idosas no } \\
\text { domicílio. } \\
\text { Autor: } \\
\text { Martins et al.; } 2007\end{array}$ & $\begin{array}{c}\text { Artigo A - Necessida- } \\
\text { des de educação em } \\
\text { saúde dos cuidadores } \\
\text { de pessoas idosas no } \\
\text { domicílio. } \\
\text { Autor: } \\
\text { Martins et al.; } 2007\end{array}$ & $\begin{array}{l}\text { Identificar e classificar } \\
\text { as necessidades de } \\
\text { saúde e educação, } \\
\text { apresentadas por } \\
\text { cuidadores de idosos, } \\
\text { baseado } \\
\text { na Classificação } \\
\text { Internacional das Prá- } \\
\text { ticas de Enfermagem } \\
\text { em Saúde Coletiva } \\
\text { (CIPESC). }\end{array}$ & $\begin{array}{c}\text { A atenção ao cuidador } \\
\text { de idosos, bem como } \\
\text { a Política Atenção } \\
\text { Integral à Saúde do } \\
\text { Idoso (PAISI), devem } \\
\text { ser legitimadas o mais } \\
\text { breve possível; que a } \\
\text { temática em estudo } \\
\text { se torne objeto de } \\
\text { processos educativos } \\
\text { e informativos } \\
\text { permanentes; } \\
\text { Promover }\end{array}$ \\
\hline $\begin{array}{l}\text { Artigo B - Grupo Feliz } \\
\text { Idade: cuidado de } \\
\text { enfermagem para a } \\
\text { promoção da saúde } \\
\text { na terceira idade. } \\
\quad \text { Autor: } \\
\text { Victor et al; } 2007\end{array}$ & $\begin{array}{l}\text { Relato de experiência } \\
\text { Amostra composta } \\
\text { por } 124 \text { idosos. }\end{array}$ & $\begin{array}{c}\text { Relatar a experiência } \\
\text { da formação de um } \\
\text { grupo de idosos e a } \\
\text { importância do cui- } \\
\text { dado de enfermagem } \\
\text { na percepção dos } \\
\text { idosos. }\end{array}$ & $\begin{array}{c}\text { Adesão crescente } \\
\text { de idosos ao grupo, } \\
\text { relatos de melhoria } \\
\text { na qualidade de vida } \\
\text { do grupo. Estratégias } \\
\text { grupais devem ser } \\
\text { estimuladas, para a } \\
\text { promoção da saúde, } \\
\text { em especial, } \\
\text { na terceira idade. }\end{array}$ \\
\hline
\end{tabular}

As dificuldades e necessidades dos cuidadores de idosos foram abordadas com excelência através dos pressupostos da Classificação Internacional das Práticas de Enfermagem em Saúde Coletiva (CIPESC). Isso resulta na importância da aplicabilidade dessa classificação não só enquanto instrumento do processo de trabalho assistencial do enfermeiro, mas também como aliado à práticas de pesquisa. O CIPESC permite visibilizar as estruturas maiores que organizam o trabalho da enfermagem, e ao mesmo tempo, pode se tornar um potencializador da avaliação processual dos resultados, benefícios e impactos da ação da enfermagem (CUBAS, EGRY; 2008).

Cabe destacar, conforme o material analisado, a carência de políticas públicas voltadas ao idoso e seu cuidador. Para isso, ações educativas que embasem as práticas empíricas dos cuidadores, que os tornem mais confiantes ao prestar o cuidar e que diminuam os níveis de estresse, são relevantes. Na obra, observa-se 
mais um indicador do quão indispensável é a participação do enfermeiro na assistência ao idoso, pois é o enfermeiro quem detém a competência de atuar como educador em saúde junto à equipe, cliente e familiares no desempenho de suas funções (Backes et al.; 2008).

O material aponta que educar trata-se de uma competência importante e inerente do trabalho do enfermeiro e requer o desenvolvimento de um pensar crítico-reflexivo. A partir disso, permite desvelar a realidade e propor ações transformadoras que direcionem o indivíduo a sua autonomia e emancipação, enquanto sujeito histórico e social capaz de sugerir e opinar nas decisões de saúde para o cuidar de si, de sua família e da coletividade (OLIVEIRA et al.; 2005).

$\mathrm{O}$ artigo B faz inferência à realização de um Grupo de Promoção à Saúde (GPS), o qual pode ser concebido como instrumento a serviço da autonomia e do desenvolvimento contínuo do nível de saúde e condições de vida. Nesses grupos, há uma intervenção coletiva e interdisciplinar de saúde, formada por um processo grupal, até o limite ético de eliminação das diferenças desnecessárias e inevitáveis entre grupos humanos (SANTOS et al.; 2006).

O GPS, quando fundamentado no amplo conceito de saúde, contempla as dimensões biopsicossociais relacionadas ao binômio saúde-doença e ao envelhecimento saudável. Dessa forma, a saúde é entendida de acordo com um conceito positivo, sendo vivenciada como de ordem natural, rompendo com a representação social da doença enquanto fatalidade.

Com a realização do GPS no estudo em questão, visualizou-se a importância desses grupos na melhoria da qualidade de vida do idoso, uma vez que não só promovem a saúde física, mas também potencializam o idoso a desenvolver uma nova maneira de se cuidar. Outro aspecto que merece ser destacado por meio do artigo B é a capacidade que esses grupos detêm de atuarem como agregadores sociais, o que instiga o idoso a manter-se em sociedade, e interagir ativamente nela.

Vasconcelos, Lima e Costa (2007) afirmam ser o envelhecer um processo de mudança e renovação das atitudes básicas que envolvem as áreas física e mental, interpessoal, relacional, afetiva e emocional. Baseia-se no desenvolvimento das potencialidades individuais, na motivação de conhecimentos e atividades sociais, incitando, dessa forma, sua capacidade de decisão. É essencial que os profissionais de saúde tomem conhecimento desse processo, compreendendo sua complexidade e magnitude, atuando em prol da promoção da saúde dos idosos (MARTINS et al.; 2007).

Em vista do exposto, evidencia-se que a enfermagem vem produzindo conhecimentos através dos trabalhos em grupo. Os grupos são espaços que favorecem o empoderamento dos indivíduos, tanto a nível individual como coletivo, 
por meio da valorização dos diversos saberes, e da possibilidade de intervenção criativa no processo saúde-doença de cada ser.

Compreender a saúde e sua promoção sensibiliza os profissionais de saúde a rever conceitos e teorias que fundamentam suas práticas. Com isso, tais profissionais vislumbram um cuidado mais próximo das reais necessidades dos usuários, e dos significados que eles têm atribuído ao processo saúde-doença que enfrentam. Com esse entendimento, a enfermagem brasileira tem se sobressaído em estudos que valorizam e integram o cuidado, as experiências, os sentimentos, os valores e as crenças dos usuários na promoção da qualidade de vida das pessoas e grupos (OLIVEIRA et al.;2009).

No tocante, a obra C, apresentada no quadro 2, propõe alicerçar o cuidado ao idoso em suas perspectivas de saúde e doença. Assim, o cuidado passa a ser sustentado em uma relação mútua, entre o cuidador e o ser cuidado. Para isso, o artigo propõe a existência de uma relação entre cultura e saúde, o que tem sido amplamente advogada por pesquisadores.

\begin{tabular}{|c|c|c|c|}
\hline Titulo do artigo & Metodologia & $\begin{array}{l}\text { Intervenção } \\
\text { estudada }\end{array}$ & $\begin{array}{l}\text { Recomendações/ } \\
\text { conclusões }\end{array}$ \\
\hline $\begin{array}{l}\text { Artigo C - Envolvi- } \\
\text { mentos da teoria do } \\
\text { cuidado cultural na } \\
\text { sustentabilidade do } \\
\text { cuidado gerontológico. } \\
\text { Autor: } \\
\text { Hammerschmidt et al.; } \\
2007\end{array}$ & $\begin{array}{c}\text { Ensaio reflexivo } \\
\text { Realizado nas base de } \\
\text { dados Lilacs, no perí- } \\
\text { odo de } 1970 \text { a } 2006, \\
\text { com as palavras- } \\
\text { chaves: } \\
\text { Cuidado Gerontológi- } \\
\text { co, Teoria da Diversida- } \\
\text { de e Universalidade do } \\
\text { Cuidado Cultural. }\end{array}$ & $\begin{array}{c}\text { Desvelar aspectos do } \\
\text { cuidado gerontológico } \\
\text { alicerçado na Teoria da } \\
\text { Diversidade e Univer- } \\
\text { salidade do Cuidado } \\
\text { Cultural com vistas a } \\
\text { subsidiar o processo } \\
\text { de cuidar digno à } \\
\text { pessoa idosa. }\end{array}$ & $\begin{array}{l}\text { Produzir } \\
\text { conhecimentos através } \\
\text { de pesquisas geronto- } \\
\text { lógicas, e que haja um } \\
\text { estímulo ao desenvol- } \\
\text { vimento de competên- } \\
\text { cias para a prática do } \\
\text { cuidado gerontológico } \\
\text { digno à pessoa idosa. }\end{array}$ \\
\hline $\begin{array}{c}\text { Artigo D - } \\
\text { Ética e estética: envol- } \\
\text { vimentos na promoção } \\
\text { do cuidado } \\
\text { gerontológico de enfer- } \\
\text { magem. } \\
\text { Autores: } \\
\text { Hammerschmidt } \\
\text { et al.; 2006. }\end{array}$ & $\begin{array}{c}\text { Estudo reflexivo } \\
\text { Realizado nas bases } \\
\text { de dados Lilacs, } \\
\text { Medline e no acervo } \\
\text { da biblioteca da } \\
\text { Universidade Federal } \\
\text { do Paraná. O período } \\
\text { pesquisado na base } \\
\text { de dados foi do ano de } \\
1990 \text { a } 2006\end{array}$ & $\begin{array}{l}\text { Levantar a produção } \\
\text { técnica-científica } \\
\text { relacionada à área da } \\
\text { saúde, em especial, } \\
\text { da enfermagem sobre } \\
\text { a ética e a estética na } \\
\text { promoção do cuidado } \\
\text { gerontológico de enfer- } \\
\text { magem }\end{array}$ & $\begin{array}{l}\text { Realizar uma "plástica } \\
\text { no cuidado } \\
\text { tradicional", com } \\
\text { a reestruturação } \\
\text { e reorganização das } \\
\text { ações de enfermagem. } \\
\text { e a necessidade } \\
\text { de reorganização do } \\
\text { cuidado gerontológico, } \\
\text { de modo a embasar e } \\
\text { preparar o atendimento } \\
\text { ao idoso, e permitir a } \\
\text { criação de um cuidado } \\
\text { de enfermagem em } \\
\text { gerontologia }\end{array}$ \\
\hline
\end{tabular}

Quadro 2. Apresentação da síntese do artigo C e D da revisão integrativa.

Sob o ponto de vista antropológico, cultura é constituída das manifestações de vida de um povo, de seu modo de pensar, sentir, agir e transmitir sua 
cultura. Essa concepção deve ser considerada no contexto saúde, almejando valorizar as manifestações culturais das pessoas diante da família, da doença e do acesso aos serviços de saúde, assim como da cura e do alívio do sofrimento (OLIVEIRA et al.;2009). Diante dessa compreensão, o cuidado, para quem o exerce e para quem ele é direcionado, deve ancorar-se no entendimento dos significados culturais dos seres envolvidos.

O uso da Teoria da Diversidade e Universalidade do Cuidado Cultural, no artigo $C$ remete à análise de que o cuidado humano é um fenômeno universal, e que as formas de cuidar variam culturalmente. Isso permite que o cuidado gerontológico flua de maneira a superar empecilhos que se geram em virtude da diversidade cultural.

Em meio a isso, o crescimento da população idosa é uma realidade, não só no Brasil, mas em todo o mundo. Isso exige dos profissionais de saúde um cuidado específico, que rejeite atitudes negativas, e que esteja baseado na busca da autonomia e da qualidade de vida, mesmo para aqueles idosos mais fragilizados (GANDOLPHO, FERRARI; 2006).

Frente a essa realidade, é urgente a adoção de pesquisas no campo gerontológico, uma vez que elas devem ser o reflexo do acelerado processo de envelhecimento populacional da atualidade, e da preocupação da classe em cuidar do idoso de maneira condizente com suas necessidades (HAMMERSCHMIDT et al.;2007). Assim, refletir sobre a pesquisa em enfermagem neste campo permite compreender a necessidade e a complexidade da relação entre saber e fazer em enfermagem, e desvenda os diversos prismas que compõem a sequência de cuidados à pessoa idosa.

Na perspectiva do cuidado gerontológico, o artigo D enfoca o aspecto ético na promoção do cuidado, valorizando a interação profissional/idoso/família. Entretanto, o estudo demonstra poucas produções científicas que contemplem essa temática, fato que dificultou a análise do material, evidenciando a necessidade de mais estudos nesta área.

A relação pessoa a pessoa é uma interação de dois ou mais seres humanos, de maneira que ambos possam compartilhar seus sentimentos, valores e significados mediante estabelecimento da comunicação (GADOLPHO, FERRARI; 2006). Essa, por sua vez, representa o fundamento cultural da pessoa humana, entretanto, trata-se de um processo que implica saber ouvir, entender e valorizar o outro.

Nessa ótica, a Enfermagem desenvolve suas ações de cuidar pautada em um processo de diálogo, valorizando a individualidade de cada ser. Assim, aqueles que se dedicam a essa profissão comprometem-se com a saúde do indivíduo e da coletividade, atuando diretamente na promoção, proteção, recu- 
peração da saúde e reabilitação das pessoas, obedecendo aos preceitos da ética e da legitimidade.

A Enfermagem é um instrumento de ação e efetivação do cuidar, sendo percebida como uma presença próxima e aberta para o ser cuidado, lidando sempre com o processo dinâmico de saúde, adoecimento e bem-estar de cada pessoa (LEITE et al.; 2009). Assim, busca integrar, coadunar, a uma visão holística do indivíduo, os princípios técnicos, científicos, sociais com a arte, a estética, a ética, a intuição e a descoberta da relação do processo de cuidar interpessoal.

\section{Considerações Finais}

Por meio da presente revisão integrativa, foi possível visualizar além das evidências de estratégias eficazes provenientes de pesquisas envolvendo o envelhecimento ativo, a necessidade de intensificar esforços, segundos os artigos analisados, como o cuidado domiciliar de idosos. Entretanto, a maior limitação do estudo, pode estar relacionada ao fato de utilizamos na busca somente os descritores DeCS para recuperar os artigos nas bases de dados eletrônicas. Constatou-se um descompasso entre esses termos e as palavras-chave utilizadas pelos autores dos artigos analisados, considerando que a expressão envelhecimento ativo não é um descritor DeCS, o que pode ter contribuído para que muitos trabalhos não tenham sido acessados.

Diante dos achados o enfermeiro, assim como os demais integrantes da equipe multidisciplinar, não deve focar seu cuidado ao idoso portador de doenças unicamente, mas atuar em uma perspectiva de promoção, educação, manutenção e recuperação desse ser. O profissional deve respeitar a independência do idoso, primando sua participação nesse processo de cuidado, o que permite que a assistência se torne qualificada, de maneira a não se ter invasão ou posse. Para que isso seja vivenciado, é preciso reconhecer que o ciclo de vida consiste em um aglomerado de etapas relacionadas entre si integradamente, segundo observamos nos estudos analisados.

Nesse sentido, a enfermagem deve fortalecer sua prática no que se refere ao cuidado gerontológico, priorizando a autonomia dos idosos, e atendimento das necessidades biopsíquicas, socioculturais e espirituais, estimulando o autocuidado, autodeterminação e a independência, de modo a manter sua capacidade e qualidade de vida. Além de assistir o idoso, o cuidado gerontológico almeja atuar conscientizando família e comunidade para aceitação do envelhecimento como parte integrante do ciclo de vida.

No estudo, é nítido que as estratégias de promoção da saúde são vitais para o envelhecimento ativo, onde a formação de Grupos de Promoção à 
Saúde, por exemplo, favorece a autonomia do ser idoso através de um cuidado específico que prioriza a qualidade de vida. Assim, enfermeiros e idosos devem atuar em uma relação interpessoal, baseada na comunicação e em princípios éticos.

Cabe destacar os vieses da pesquisa, essencialmente no que concerne à ética no cuidado gerontológico. Assim, o contexto ético na enfermagem abrange comportamentos e ações que envolvem conhecimento, valores, habilidades e atitudes depreendidas na acepção de favorecer as potencialidades do ser humano, visando manter ou melhorar a condição humana no processo de viver e morrer. É crucial que a assistência seja fundamentada nos princípios da ética e da legalidade, entretanto cabe ressaltar que a ética ainda é difícil de ser vivenciada, disseminada e divulgada na prática, segundo os materiais analisados, uma vez que muitos valores estão envolvidos em contextos culturais, sociais e religiosos, e ainda aqueles relacionados ao caráter do indivíduo/profissional.

Apesar das publicações estarem aumentando nos últimos anos, no que se refere aos estudos sobre envelhecimento ativo, faz-se necessária a realização de outras pesquisas na área de enfermagem. Essas poderão ressignificar a produção científica já produzida, adequando-a aos inúmeros cenários de cuidado de enfermagem, visto que segundo esta revisão integrativa, há poucos estudos específicos na área, incluindo estudos interdisciplinares que envolvam a profissão.

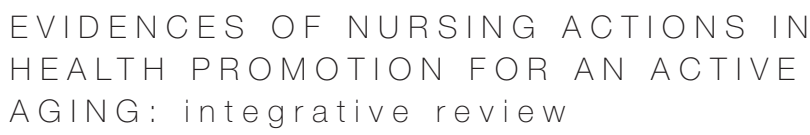

Active Ageing is the process of optimizing the health opportunities for health, with the aim of increasing the elderly life quality. For the nurse, member of the multidisciplinary team, to promote a specific geriatric care constitute a primordial activity. This integrative review aimed to analyze the literature available evidences about the actions of Nursing Health Promotion for an Active Aging. To the sample selection the databases Lilacs, Medline, Cochrane, Cinahl and Scopus were consulted, and the total of four articles constituted the sample. The studies emphasize that aspects of health promotion for the elderly are essential for active aging and suggest the existence of a relationship between culture and health. The present review aid the 

interpersonal relationship between the nurse and the elderly, based on communication and ethical principles

keywords

Nursing. Elderly. Health Promotion.

\section{referências}

BACKES, Vânia Marli Schubert; LINO, Mônica Motta; PRADO, Marta Lenise; REIBNITZ Kenia Schimidt; CABAVER, Bruna Pedroso. Competência dos enfermeiros na atuação como educador em saúde. Revista Brasileira de Enfermagem, Rio de Janeiro, v. 61, n. 6, Nov/Dez, 2008.

BRASIL. Ministério da Previdência e Assistência Social. Secretaria de Assistência Social. - Politica Nacional do Idoso. Lei n ${ }^{\circ} 8.842$ de 4 de janeiro de 1994. Decreto n¹.948 de 3 de julho de 1996. 2 ed. Brasilia: MPAS, 1998.

CAMARANO, Ana Amélia. "Envelhecimento da população brasileira: uma contribuição demográfica". In: FREITAS, E. V. de et al. Tratado de geriatria e gerontologia. Rio de Janeiro: Guanabara, 2002. Cap.6, p.58-71.

CUBAS, Márcia Regina; EGRY, Emiko Yoshikawa. Classificação Internacional de Práticas de Enfermagem em Saúde Coletiva - CIPESC. Revista da Escola de Enfermagem da USP, São Paulo, v. 42, n. 1, mar, 2008.

GADOLPHO, Maria Ângela; FERRARI, Maria Auxiliadora. A enfermagem cuidando do idoso: reflexões bioéticas. O mundo da saúde, São Paulo, v. 30, n. 3, Jul/Set, 2006.

GALVÃO, Cristina Maria; SAWADA, Namie Okino; ROSSI, Lídia Aparecida. A prática baseada em evidências: considerações teóricas para sua implementação na enfermagem perioperatória. Revista Latino-amenicana de Enfermagem, Ribeirão Preto, v. 10, n. 5, Set/Out, 2002

HAMMERSCHMIDT, Karina Silveira de Almeida; BORGHI, Ângela Cristina da Silva; LENARDT, Maria Helena. Pesquisas de enfermagem em gerontologia. Cogitare Enfermagem, Curitiba, v. 12, n. 2, Abr/Jun, 2007.

HAMMERSCHMIDT, Karina Silveira de Almeida; ZAGONEL, Ivete Palmira Sanson; LENARDT, Maria Helena. Envolvimentos da teoria do cuidado cultural na sustentabilidade do cuidado gerontológico. Acta Paulista de Enfermagem, São Paulo, v. 20, n. 3, Set. 2007.

HAMMERSCHMIDT, Karina Silveira de Almeida; BORGHI, Ângela Cristina da Silva; LENARDT, Maria Helena. Ética e estética: envolvimentos na promoção do cuidado gerontológico de enfermagem. Texto \& Contexto - Enfermagem, Florianópolis, v. 15, n. spe, 2006. Disponível em: http://www.scielo.br/scielo.php?script=sci_arttext\&pid=S010407072006000500013\&lng=en\&nrm=iso. Acesso em : 31 de maio de 2010.

LEITE, Alice lana Tavares; GOMES E CLAUDINO Hellen; SANTOS, Sérgio Ribeiro. A importância de ser ético: da teoria à prática na enfermagem. Cogitare Enfermagem, Curitiba, v. 14, n. 1, Jan/Mar, 2009.

LUNARDI, Valéria L. et al. Impacto dos resultados das pesquisas em enfermagem na prática profissional. Cogitare Enfermagem, Curitiba, v. 14, n. 1, Jan/Mar, 2009.

MARTINS, Josiane de J. et al. Educação em saúde como suporte para a qualidade de vida de grupos da terceira idade. Revista Eletrônica de Enfermagem, Goiânia, v. 9, n. 2, Mai/Ago, 2007 
MARTINS, Josiane de Jesus et al . Necessidades de educação em saúde dos cuidadores de pessoas idosas no domicílio. Texto \& contexto - Enfermagem, Florianópolis, v. 16, n. 2, June 2007. Disponível em: http://muw.scielo.br/scielo.php?script=sci_ arttext\&pid=S0104-07072007000200007\&lng=en\&nrm=iso. Acesso em: 31 de Maio de 2010.

OLIVEIRA, Márcia M. C. et al. Análise das investigações em enfermagem e o uso da teoria do cuidado cultural. Ciência, Cuidado e Saúde,Maringá, v. 8, n. 1, Jan/Mar, 2009.

OLIVEIRA, Márcia M. C. et al. Aplicação do processo de relação interpessoal de Travelbee com mãe de recém-nascido internado em uma unidade neonatal. Revista da Escola de Enfermagem da USP, São Paulo, v. 39, n. 4, Dez, 2005.

PICCINI, Roberto Xavier et al. Necessidades de saúde comuns aos idosos: efetividade na oferta e utilização em atenção básica à saúde. Ciência \& Saúde Coletiva, Rio de Janeiro, vol.11, n.3, Jul/Set, 2006.

SANTOS, Silvana S. et al. Promoção da saúde da pessoa idosa: compromisso da enfermagem gerontogeriátrica. Acta Paulista de Enfermagem, São Paulo, v. 21, n. 4, 2008. Disponível em: http://wnw.scielo.br/scielo.php?script=sci_arttext\&pid=S010321002008000400018\&lng=en\&nrm=iso\&tlng=pt .

SANTOS, Luciane de M. et al. Grupos de promoção à saúde no desenvolvimento da autonomia, condições de vida e saúde. Revista de Saúde Pública, São Paulo, v. 40, n. 2, Abr, 2006.

URSI, Elizabeth Silva. Prevenção de lesões de pele no perioperatório: revisão integrativa da literatura. 2005. 130 f. Dissertação (Mestrado em Enfermagem) - Escola de Enfermagem de Ribeirão Preto, Universidade de São Paulo, Ribeirão Preto. 2005.

VASCONCELOS, Kelly R. B.; LIMA, Narúbia A.; COSTA, Kemle S. Envelhecimento ativo na visão de participantes de um grupo de terceira idade. Fragmentos de Cultura, Goiânia,v. 17, n. 3/4, Mar/Abr, 2007.

VICTOR, Janaína Fonseca et al. Grupo Feliz Idade: cuidado de enfermagem para a promoção da saúde na terceira idade. Revista da Escola de Enfermagem da USP, São Paulo, v. 41, n. 4, Dec. 2007. Disponível em: http://mww.scielo.br/scielo. php?script=sci_arttext\&pid=S0080-62342007000400026\&lng=en\&nrm=iso . Acesso em: 31 de Maio de 2010.

WONG, Laura L. Rodríguez; CARVALHO, J. A. O rápido processo de envelhecimento populacional do Brasil: sérios desafios para as políticas públicas. Revista Brasileira de Estudos de População. São Paulo, v. 23, n. 1, Jan/Jun, 2006. Disponível em: http:// www.scielo.br/scielo.php?script=sci_arttext\&pid=S0102-30982006000100002\&lng= en\&nrm=iso. Acesso em: 31 de Maio de 2010.

WORLD HEALTH ORGANIZATION. Envelhecimento ativo: uma política de saúde / World Health Organization; tradução Suzana Gontijo. - Brasília: Organização Pan-Americana da Saúde, 2005. 60p. Disponível em: http://portal.saude.gov.br/portal/arquivos/pdf/ envelhecimento_ativo.pdf

Recebido: 15-04-2010

$1^{\text {a }}$ Revisão: 14-06-2010

$2^{a}$ Revisão: 18-07-2010

Aceite Final: 01-10-2010 